\title{
APTITUDES COGNITIVAS Y ESTRATEGIAS DE MEMORIZACIÓN SECUENCIAL DE LOS ALUMNOS DE UN CENTRO EDUCATIVO PRIVADO DE COMAS
}

\author{
COGNITIVE APTITUDES AND STRATEGIES OF SEQUENTIAL \\ MEMORIZATION OF PUPILS IN A PRIVATE SCHOOL IN COMAS
}

\author{
Pedro Garcia P. ${ }^{1}$, Victoria llaja R. ${ }^{1}$, César Sarriá J. ${ }^{1}$, Jorge Soto M ${ }^{1}$, Marina Salazar C. ${ }^{1}$, Julio Alvites R. ${ }^{1}$ \\ Universidad Nacional Mayor de San Marcos, Lima, Perú \\ (RECIBIDO EL 9/02/2009, ACEPTADO EL 17/06/2009)
}

\begin{abstract}
RESUMEN
En el momento actual se insiste en considerar la importancia de los periodos críticos del desarrollo del niño, refiriéndose a toda la influencia de las condiciones sociales, educativas y familiares durante todo el proceso de formación, las que a su vez permiten la adquisición y desarrollo tanto de procesos cognoscitivos, afectivos, como conativo-motivacionales y en líneas generales de su personalidad como un todo.

Los estudios de la memorización, por tratarse de un proceso cognoscitivo indispensable en el aprendizaje, que permite el almacenaje y actualización de la información, son y serán siempre de gran trascendencia. Asimismo, independientemente de la estrategia de memorización que se utilice, este proceso ayuda a estructurar las aptitudes cognitivas en cada etapa de desarrollo cognitivo del niño.

Una evaluación oportuna tanto de las estrategias de memorización como de las aptitudes, nos permite la detección posterior de problemas de aprendizaje escolar, sean éstos de naturaleza global o específica.

De allí que en este estudio se pretende describir y explicar cómo son las estrategias de memorización más frecuentes en los niños de 6 a 11 años que coadyuvan favorablemente o inadecuadamente en la estructuración del componente intelectivo, o de las aptitudes cognitivas en la niñez.
\end{abstract}

Palabras clave: Memoria secuencial, aptitudes cognitivas, estrategias de memorización, sistema de la personalidad.

\footnotetext{
ABSTRACT

In the current moment it is insisted on considering to be the importance of the critical period of the development of the child, referring to the whole influence of the social, educational and familiar

1 Docentes investigadores del Instituto de Investigaciones Psicológicas de la Facultad de Psicología, Universidad Nacional Mayor de San Marcos, Lima-Perú. E-mail: pgarciap@hotmail.com
} 
conditions during the whole process of training, which in turn allow the acquisition and develop so much of cognitive, affective processes, since as conative-motivational and in general lines of your personality as an everything.

The studies of the memorization, for treating itself about a cognitive indispensable process in the learning, which allows the storage and update of the information, are and they will be always of great transcendency. Likewise independently of the strategy of memorization that is in use, this one I try help to constructing the cognitive aptitudes in every stage of cognitive development of the child.

An opportune evaluation so much of the strategies of memorization, since as of the aptitudes, allows us the later detection of problems of school learning, be these of global or specific nature.

Of there that in this study it tries to describe and to explain like are the most frequent strategies of memorization in the children from 6 to 11 years that they contribute favourably or inadequately in the structure of the component intellective, or of the cognitive aptitudes in the childhood.

Keywords: Sequential memory, cognitive aptitudes, strategies of memorization, system of the personality.

\section{INTRODUCCIÓN}

Además de la alta incidencia y la frecuencia de los problemas de aprendizaje en la primera etapa escolar, no existe en el medio estudios que evalúen un perfil integral de memoria secuencial, y su relación con la adquisición de las aptitudes intelectivas de los niños de 6 a 11 años. Tampoco se cuenta con instrumentos validados y baremados que evalúen de manera confiable e integral el perfil de memoria secuencial basada en la mediación verbal y el desarrollo de las aptitudes cognitivas de esta etapa de la niñez. De allí que consideramos de suma utilidad la evaluación de estos procesos y su explicación bajo la propuesta del modelo del sistema de la personalidad.

\section{Estudios previos}

La Prueba de Memoria de Colores, que evalúa memoria secuencial, ha mostrado ser útil en la identificación de diferentes tipos de problemas de memoria, en niños discapacitados para aprender y con déficit de atención. El perfil que se obtiene de las cuatro subpruebas permite al examinador interpretar las estrategias preferidas del niño para retener información en la memoria. Al identificar las fuerzas y debilidades del niño en su perfil de memoria, se puede determinar si el aprendizaje y la atención pueden ser mejorados por la presentación visual versus verbal de materiales, y si el niño debe dar una respuesta motora o verbal.

Hay varios reportes de investigación sobre la Prueba de Memoria de Colores:

- Lindgren \& Richman (1984). "Immediate Memory Functions of Verbally Deficit Reading Disabled Children, Journal of Learning Disabilities, 17, 222-225. Este estudio comparó los hallazgos de la Prueba de Memoria de Colores de niños de 8 a 12 años discapacitados en la lectura con un grupo control normal, emparejados por edad. Los resultados indican que los discapacitados en lectura de menos edad tuvieron dificultad tanto con la memoria verbal como con el modelo de memoria cruzada (visual-verbal), 
pero tuvieron aún una adecuada memoria en el material presentado visualmente con respuesta motora. Los niños DA de más edad sólo tuvieron dificultades en la memoria verbal y una adecuada memoria intermodal y visual. Se discuten las implicaciones para la instrucción en la lectura y lenguaje.

- Lindgren, Richman \& Eliason (1986). Memory processes. Reading Disability Subtypes, 2, 173-1819. Este estudio examinó los resultados de la Prueba de Memoria de Colores en 3 subgrupos de DA (desorden de lenguaje, desorden visoperceptual y desorden de memoria), emparejados por edad y CI. Se identificaron perfiles específicos de memoria para cada subgrupo; y los variados perfiles se basaron en el modo de la respuesta o de la presentación del estímulo (verbal-visual). Los datos indican que es posible identificar estrategias de memoria preferidas, que tienen implicaciones para elegir el tipo de instrucción de lectura que debe ser apropiado para los subgrupos de niños discapacitados en lectura.

- Wood, Richman \& Eliason (1989). "Inmediate memory functions in reading disability subtypes”. Brain and Language, 36, 181-192. Este estudio examinó los perfiles de memoria en la Prueba de Memoria de Colores para tres grupos de discapacidades en la lectura (desorden perceptual-motor, desorden verbal, desorden de memoria), emparejados por edad y CI. Los datos intercorrelacionados demostraron claramente que los factores verbal y visual pueden estar reparados. Los resultados indican que los niños discapacitados en lectura, prescindiendo del subtipo, experimentan algún tipo de dificultad en la memoria. Sin embargo, algunos tienen solo una dificultad de producción, que permite la enseñanza de estrategias de lectura y memoria alternativa.

Otros estudios de memoria verbal efectuados con el Test de Recuerdo Selectivo de Buschke \& Altman, son los realizados por Llaja,V. (1983, 2000) quien efectuó un estudio piloto sobre el Síndrome de Amnesia Global Transitoria donde se obtuvieron conclusiones, estableciendo un diagnóstico diferencial con otras entidades nosológicas. Uno de los hallazgos más relevantes fue que, a través del Test, se pudo diferenciar el desempeño amnésico de pacientes con amnesia global transitoria cuya etiología fue psicógena, y aquellos que tenían patología estructural, tipo isquemia cerebral transitoria. Lo característico en este síndrome es que no dejaba secuela, a excepción del episodio de amnesia, y algunos pacientes presentaron más de un episodio recurrente de amnesia. Este síndrome no es muy frecuente entre la casuística de enfermedades neurológicas.

De otro lado, se trabajó con este mismo instrumento (Llaja et.al.) en niños de 5 años donde se relacionó los estímulos lingüísticos familiares: elaborados y restringidos y la memoria verbal, en el trabajo intitulado Los estímulos lingüísticos familiares y la memoria verbal en niños de 5 años. Tesis para optar el grado de Maestra en Psicología.

Dentro de las categorías que se evaluaron, las conclusiones más relevantes fueron que existían diferencias significativas entre los niños en cuanto a su desempeño de la memoria verbal.

Las diferencias que se encontraron en las categorías de memoria a corto plazo y largo plazo pusieron de manifiesto que aquellos niños que eran estimulados con códigos elaborados tenían mejor desempeño que los del grupo comparativo, de códigos restringidos. 
La mayor diferencia se produjo en los procesos de consolidación mnésica y reproducción a largo plazo. No existía influencia de la variable sexo en el desempeño, mas sí en la variable nivel de instrucción (estos estudios obran en la biblioteca de la Universidad San Martín de Porres).

Hay un acuerdo en el campo de la Neuropsicología, que los estudios de la memoria son y serán siempre de gran trascendencia, ya que es una de las formas de actividad psíquica más importantes.

Es importante hacer hincapié que en las dos últimas décadas prácticamente se definieron dos conceptos fundamentales en la teoría de la memoria; autores como Exner, Ebbinghaus, Wernicke, Korsakoff, Bechterev \& James, entre otros, fueron los que aportaron estudios de correlación de las lesiones del lóbulo temporal y las teorías de procesamiento de la memoria, en el neocortex cerebral; surgiendo conceptos como: memoria primaria y secundaria, que permanecen hasta la actualidad.

En su versión tradicional, la memoria es definida como el curso espaciotemporal de la información en el cerebro, que comprende procesos de codificación y utilización de la información almacenada y consolidada (Ortiz, 2004).

Para Ervin y Anders (citado en Ortiz et al.), la memoria tiene cuatro sistemas de almacenaje de la información: sensorial, primario, secundario y terciario, cada uno de ellos con características bien definidas, y ya descritas anteriormente.

Toulving (1972, citado por Ortiz et al.) introdujo por primera vez la diferenciación entre memoria semántica y memoria episódica; la primera se refiere al sistema para recibir, retener y transmitir información acerca del significado de las palabras, los conceptos y su clasificación, y la segunda es la memoria para experiencias personales y sus relaciones temporales. A pesar de esta revisión, prevalece la primera diferenciación de James sobre memoria primaria y secundaria como la fundamental.

Sin embargo, todos estos planteamientos han sido analizados críticamente en las obras de Ortiz, P. (1994, 1997, 1998, 1999, 2002, 2004), el autor ha sugerido que es necesario un replanteamiento del problema de la memoria dentro de un modelo que tome en cuenta la integridad de la persona y la conciencia.

El concepto de memoria y los demás conceptos psicobiológicos deben ensamblarse en un modelo único, a fin de superar las hipótesis ad hoc que se introducen para explicar cada tipo, estructura, proceso o módulo de la actividad psíquica.

Gran parte de este modelo es descrito y aplicado a la explicación de las aptitudes cognitivas y las estrategias de memorización, materia del presente estudio.

Nos hemos planteado el siguiente problema de investigación ¿Cuál es la relación existente entre las aptitudes cognitivas y las estrategias de memorización secuencial de los alumnos de un Centro Educativo Privado de Comas?

Para analizar las aptitudes cognitivas y las estrategias de memorización, queremos asumir el modelo teórico basado en el Sistema de la Personalidad, del Dr.Ortiz, P. (20-21). Para él, cada individuo humano es una personalidad y cada personalidad tiene: 1) sus 
niveles o formas de organización, 2) su estructura y sus componentes estructurales, 3) su actividad y sus planos de actividad, 4) sus procesos de determinación, 5) sus procesos formativos, 6) su actuación y estrategias de actuación concreta y 7) sus atributos y capacidades.

Debemos aclarar, sin embargo, que esta forma de concebir la organización de la personalidad, de ninguna manera quiere decir que está formada por estratos o pisos superpuestos. Por eso al decir que todo el individuo humano es celular, que todo el individuo es tisular, que todo el individuo es un organismo, que todo el individuo es un psiquismo, ya es más fácil entender porque Ortiz (ob. cit.) dice que todo el individuo es una personalidad.

El individuo humano es una personalidad cuya estructura y actividades dependen de la información psíquica consciente que está codificada en las redes nerviosas del neocórtex cerebral. Este mayor nivel de organización de la persona depende del sistema de la conciencia que comprende a su vez tres componentes o subsistemas: afectivo- emotivo, cognitivo-productivo y conativo-volitivo.. Por ende, el sistema de la personalidad está constituido por tres componentes: que son el temperamento, el intelecto y el carácter. Ahora se puede decir que corresponden a la emoción, la cognición, y la motivación, y que cada uno de estos componentes de la conciencia es la base de desarrollo de los respectivos componentes de la personalidad; cada uno de estos componentes depende de la clase de información psíquica que ha servido de base de su desarrollo durante las etapas formativas de la infancia, la niñez y la adolescencia respectivamente (20-21).

Así, el temperamento es el componente de la personalidad que comprende toda la estructura interna del individuo, que se organiza desde el componente afectivo-emotivo de la conciencia. Es una forma de actividad ligada en principio a necesidades internas y su satisfacción consciente dentro de los procesos de esta sociedad. Se expresa por los gestos que conforman el comportamiento por medio del cual uno establece sus relaciones interpersonales. Su soporte funcional son las áreas órbito-frontal del neocórtex.

El intelecto de la personalidad es la estructura de la persona cuya actividad externa se relaciona con la actividad social productiva, una relación básicamente laboral, que se expresa por medio de las acciones que constituyen el desempeño personal. Cuyo soporte funcional son las áreas parieto-temporo-occipital del neocórtex (áreas intrínsecas posteriores).

Finalmente, Ortiz considera que el carácter es el componente ético de la personalidad. El primer nivel de este componente corresponde a la actividad consciente que se organiza en base a la información del sistema conativo-volitivo cuyo soporte funcional es el área dorso lateral del neocórtex prefrontal.

El carácter se expresa en los actos de la conducta por medio de la cual cada personalidad se relaciona con las demás al interior de las relaciones económicas de sociedad.

En lo que se refiere a los tres sistemas ya mencionados más arriba, el Sistema afectivoemotivo podemos verlo como estructura cuando hablamos de disposiciones afectivas, estados de ánimo, de humor, o como actividad cuando hablamos de emociones y gestos emocionales. En el sistema cognitivo-productivo, la estructura de este sistema aparece como el conjunto de aptitudes cognitivas, es decir, el conjunto de capacidades que llamamos 
conocimientos, destrezas y habilidades. En cuanto al sistema conativo-volitivo, es un sistema característico, único y exclusivo del hombre; de este componente de la conciencia depende que el accionar de los hombres se haya convertido en conducta, es decir, en una forma de actuación personal en la que se integran el comportamiento emotivo y desempeño productivo en base a reglas morales que traducen la estructura ética de la sociedad.

La información psíquica conativa viene a ser los motivos y valores que son usados como reglas o procedimientos de decisión volitiva, por medio de los cuales se organiza la actividad autoconsciente de la personalidad, la persona se autovalora y es consciente de sí misma y de las consecuencias sociales de sus actos.

Las clases de información que llamamos motivos, como son, principalmente, las convicciones, expectativas, intereses, aspiraciones, intenciones, pasiones, ideales, objetivos, propósitos, prejuicios, perspectivas, pretensiones, responsabilidades, obligaciones, deberes, en el curso de la actividad consciente adoptan la forma de valores, y en base a éstos se organizan sus procesos volitivos (20-21).

Desde esta perspectiva teórica, asumiremos el análisis cualitativo de las aptitudes cognitivas y las estrategias de memorización en los niños de un colegio privado de Comas, tratando de establecer una aproximación objetiva a las características del perfil cognitivo y su relación con las estrategias de memoria. Aspiramos tener una explicación aproximada acerca de las estrategias de memorización secuencial de niños de condición socioeconómica emergente, determinar la relación que existe entre las aptitudes cognitivas y las estrategias de memorización secuencial de los niños de 6 a 11 años, y establecer los déficit de las aptitudes cognitivas, que puedan repercutir en las estrategias de memorización y, por ende, en el aprendizaje de los niños de estas edades.

Nuestras hipótesis son las siguientes:

1. Existe relación directa entre las aptitudes cognitivas y las estrategias de memorización secuencial de los niños de 6 a 11 años.

2. Existe relación directa entre las aptitudes cognitivas y las estrategias de memorización secuencial, con mediación verbal, de los niños de estas edades.

3. Existe relación directa entre las aptitudes cognitivas y las estrategias de memorización secuencial, sin mediación verbal, de los niños de estas edades.

4. Existe relación directa entre los déficits en las aptitudes cognitivas y las estrategias de memorización secuencial, con mediación verbal, de los niños de estas edades.

5. Existe relación directa entre los déficit en las aptitudes cognitivas y las estrategias de memorización en sus distintas modalidades, y las dificultades en el rendimiento académico de los niños de estas edades.

\section{Problema}

Nos planteamos el siguiente problema de investigación: ¿Cuál es la relación existente entre las aptitudes cognitivas y las estrategias de memorización secuencial de los alumnos de un Centro Educativo Privado de Comas? 


\section{Ex Hipótesis}

Existe relación directa entre las aptitudes cognitivas y las estrategias de memorización secuencial de los niños de 6 a 11 años.

\section{MÉTODO}

Es un trabajo de tipo descriptivo, correlacional, transversal y retrospectivo.

La muestra estuvo constituida por todos los niños en edad escolar, del primer grado hasta el primero de secundaria, que estudian en un Centro Educativo Privado de Comas, que fueron evaluados en el periodo comprendido de diciembre 2006 a enero 2007. La muestra fue recogida de manera intencional. Se administró la Prueba de Memoria de Colores (Lindgren \& Richman,1984) y la Prueba de Habilidad Intelectual (Factor G de inteligencia) para niños.

\section{Procesamiento estadístico}

Se utilizó el análisis estadístico SPSS, versión 15 y versión 13, para comparar frecuencias de la información demográfica de este estudio. Igualmente se calculó medidas no paramétricas para establecer si existen correlaciones directas entre la variable independiente y dependiente de nuestro estudio.

\section{Muestra}

Los niños de la muestra provienen de un centro educativo privado, ubicado en el Cono Norte de Lima Metropolitana. Se halla en la zona urbana y en un ambiente en que la actividad comercial es creciente. Los niños que asisten viven en los alrededores del colegio.

La Tabla N. ${ }^{\circ} 1$ expone la descripción demográfica relevante a los niños y sus familias. La edad promedio del grupo total es 8.2 años $(m=102$ meses, d.e. $=25.5)$, y desde 59 hasta 159 meses (4 hasta 14 años). Entre los niños (en meses, $m=100.3$, d.e. $=24.6$ ) y niñas (en meses, $\mathrm{m}=103$, d.e. $=26.2$ ) las edades fueron muy similares.

Dentro de la instrucción superior de las madres, 12 de ellas tuvieron educación técnica certificada y 4 estudios universitarios; para los padres 20 son educación técnica y 5 en nivel universitario. 
Tabla N. ${ }^{0}$ 1. Caracterización demográfica de la muestra.

\begin{tabular}{|c|c|c|c|c|c|}
\hline & & $\mathbf{N}$ & $\%$ & & \\
\hline \multicolumn{6}{|c|}{ Grado escolar } \\
\hline & Inicial (4 y 5 años) & 7 & 8.8 & & \\
\hline & Primaria (1ro al 6to) & 68 & 85.0 & & \\
\hline & Secundaria (1ro y $2 \mathrm{do}$ ) & 5 & 6.3 & & \\
\hline \multicolumn{6}{|l|}{ Sexo } \\
\hline & Varón & 33 & 41.3 & & \\
\hline & Mujer & 47 & 58.8 & & \\
\hline \multicolumn{6}{|c|}{ Familia } \\
\hline \multicolumn{6}{|c|}{ Condición civil } \\
\hline & Casados & 56 & 70.0 & & \\
\hline & Convivientes & 5 & 6.3 & & \\
\hline & Separados & 9 & 11.3 & & \\
\hline & Madre soltera & 9 & 11.3 & & \\
\hline & Viudez & 1 & 1.3 & & \\
\hline \multicolumn{6}{|c|}{ Ingresos } \\
\hline & $>1500$ & 38 & 47.5 & & \\
\hline & $1001-1500$ & 27 & 33.8 & & \\
\hline & $500-1000$ & 14 & 17.5 & & \\
\hline & $<500$ & 1 & 1.3 & & \\
\hline \multicolumn{6}{|c|}{ Los apoderados } \\
\hline & & \multicolumn{2}{|c|}{ Madre } & \multicolumn{2}{|c|}{ Padre } \\
\hline & & $\mathrm{N}$ & $\%$ & $\mathrm{~N}$ & $\%$ \\
\hline & \multicolumn{5}{|l|}{ Instrucción } \\
\hline & Secundaria & 64 & 80.0 & 55 & 68.8 \\
\hline & Superior & 16 & 20.0 & 25 & 31.3 \\
\hline \multicolumn{6}{|c|}{ Ocupación } \\
\hline & Trab. empleado & 23 & 28.8 & 39 & 48.8 \\
\hline & Trab. obrero & 10 & 12.5 & 23 & 28.8 \\
\hline & Act. Indep. & 47 & 58.8 & 18 & 22.5 \\
\hline Total & & 80 & & & \\
\hline
\end{tabular}

\section{Criterios de inclusión}

Niños con inteligencia normal

Niños con edad de 6 a 11 años.

Niños de sexo masculino y femenino.

Niños sin déficit neurológicos ni sensoriales. 


\section{Criterios de exclusión}

Niños con diagnósticos establecidos neurológicos o psiquiátricos.

Niños con problemas específicos del aprendizaje ya diagnosticados.

\section{Procedimiento}

La confiabilidad será examinada por el método de consistencia interna y se aplicará el coeficiente alfa de Cronbach (Cronbach, 1951).

La asociación entre las variables memoria y aptitud cognitiva general será examinada por la correlación lineal de Pearson (Cohen, 2001). Sin embargo, para examinar apropiadamente esta relación, se harán ajustes a las estimaciones de las correlaciones. El ajuste controlará estadísticamente el efecto de la variable edad y, por otro lado, se corregirán efectos de atenuación por el grado de confiabilidad de los instrumentos. Como se ve en la Tabla N. ${ }^{\circ}$ 2 , las estimaciones de confiabilidad están alrededor de 0.65 , y estos niveles son apenas satisfactorios sobre el error de medición relacionado con las correlaciones inter-ítem. Por lo tanto, se aplicará la correlación parcial y una corrección por atenuación a esta correlación parcial; el método está descrito en varias fuentes (Chen \& Popovich, 2002; Osborne, 2003; Bohrnstedt, 1983) que resaltan la vulnerabilidad de las estimaciones correlacionales ante el impacto de la confiabilidad de las mediciones y de la influencia de factores extraños, que en nuestra investigación es la edad.

Tabla N. ${ }^{\circ}$ 2. Estadísticos descriptivos y consistencia interna.

\begin{tabular}{|c|c|c|c|c|c|c|c|}
\hline & \multirow{2}{*}{$\begin{array}{c}\text { Alfa } \\
\text { (muestra } \\
\text { total) }\end{array}$} & \multicolumn{2}{|c|}{ Varón } & \multicolumn{2}{|c|}{ Mujer } & \multicolumn{2}{|c|}{ Total } \\
\hline & & M & $\mathrm{DE}$ & M & $\mathrm{DE}$ & M & $\mathrm{DE}$ \\
\hline Visual-Visual & 0.73 & 4.73 & 1.9 & 4.64 & 1.9 & 4.68 & 1.9 \\
\hline Visual-Verbal & 0.64 & 5.15 & 1.6 & 5.09 & 1.5 & 5.11 & 1.6 \\
\hline Verbal-Visual & 0.59 & 6.27 & 1.4 & 6.02 & 1.5 & 6.13 & 1.4 \\
\hline Verbal-Verbal & 0.61 & 6.67 & 1.4 & 6.45 & 1.4 & 6.54 & 1.44 \\
\hline Aptitudes Cog. & 0.75 & 22.30 & 6.065 & 23.02 & 5.451 & 22.73 & 5.6 \\
\hline
\end{tabular}

\section{RESULTADOS}

\section{Diferencias demográficas}

En primer lugar, las varianzas entre varones y mujeres no fueron grandes como para afectar la interpretación y aplicación de las técnicas paramétricas (F de Levene $<1.03$ ); esto sugiere que la variabilidad de los puntajes entre varones y mujeres es similar para fines prácticos y descriptivos, y las diferencias son pequeñas. De otro lado, las diferencias entre los puntajes promedio de varones y mujeres no fueron en ninguna situación estadísticamente 
significativos, tal como lo indica la prueba t de Student ( $\mathrm{p}>0.05)$. Ya que si aparecen diferencias, éstas son insignificantes (ver la Tabla N. ${ }^{\circ}$ ), por lo que los datos serán tratados como una sola unidad.

También se puede observar en la Tabla N. ${ }^{\circ} 3$ que las diferencias entre los tipos de memoria evaluados han seguido un patrón reconocible para el input visual y el verbal. En general, los puntajes de las escalas Visual-Visual y Visual-Verbal tienden a ser menores que los puntajes de las escalas Verbal-Visual y Verbal-Verbal; y los puntajes bajo y alto extremos corresponden a Visual-Visual y Verbal-Verbal, respectivamente.

Tabla N. ${ }^{\circ}$ 3. Correlaciones Pearson entre los puntajes de memoria, aptitud y edad.

\begin{tabular}{lcccccc}
\hline & $\begin{array}{c}\text { Edad } \\
\text { meses }\end{array}$ & $\begin{array}{c}\text { Visual- } \\
\text { Visual }\end{array}$ & $\begin{array}{c}\text { Visual- } \\
\text { Verbal }\end{array}$ & $\begin{array}{c}\text { Verbal- } \\
\text { Visual }\end{array}$ & $\begin{array}{c}\text { Verbal- } \\
\text { Verbal }\end{array}$ & $\begin{array}{c}\text { Aptitud } \\
\text { Cognitiva }\end{array}$ \\
\hline Edad meses & 1 & & & & & \\
Visual-Visual & $.501^{* *}$ & 1 & & & & \\
Visual-Verbal & $.313^{* *}$ & $.537^{* *}$ & 1 & & & \\
Verbal-Visual & $.321^{* *}$ & $.539^{* *}$ & $.423^{* *}$ & 1 & & \\
Verbal-Verbal & $.263^{*}$ & $.448^{* *}$ & $.327^{* *}$ & $.657^{* *}$ & 1 & \\
Aptitud Cognitiva & $.548^{* *}$ & $.405^{* *}$ & $.238^{*}$ & $.310^{* *}$ & $.334^{* *}$ & 1 \\
\hline
\end{tabular}

${ }^{* *} p<0.01 ;{ }^{*} p<0.05$

\section{DISCUSIÓN}

En base a los hallazgos estadísticos, no se observan diferencias significativas entre los puntajes obtenidos por género, lo que es similar a los resultados obtenidos en otros estudios de Memoria verbal con el Test de Recuerdo selectivo, en niños de 5 años, cuyos estímulos lingüísticos familiares fueron restringidos y elaborados (Llaja,V. 2000), por lo que en nuestro estudio se corrobora que la variable sexo no influencia en el desempeño de la memoria de secuencias.

En lo que se refiere a la puntuación obtenida en cada uno de los subtest, es notorio destacar la predominancia de lo verbal sobre lo visual ("no verbal"). Ya que si bien en el proceso de desarrollo cognoscitivo lo sensorial predomina sobre lo racional; la recodificación lingüística aparece como predominante en toda la actividad psíquica. Así mismo, Evans (1987) sostiene que existe una interdependencia e incluso habla de una equivalencia entre el lenguaje y las habilidades cognoscitivas, entre ellas la memoria. Así tenemos que varios investigadores, entre ellos Baddeley \& Patterson, resaltan la importancia del lenguaje en los procesos de memorización, lo que se ve reflejado en el Rehearsal de información que facilita la consolidación de los datos en los sistemas de memoria a largo plazo, que es predominantemente verbal, aun cuando el estímulo sea visual, mecanismo que es indispensable para incrementar la resistencia del dato sensorial o huella a la interferencia. 
Estos hallazgos cobran vigencia en un país multilingüe donde los niños bilingües presentan desventaja lexical en comparación a los niños monolingües.

Los hallazgos en la Tabla N. ${ }^{\circ} 4$ nos indican una correlación positiva entre la edad de los niños, la Memoria visual-visual y la Inteligencia "No verbal" cuando no se controló la edad, destacando una mayor correlación (de grado moderado) en el subtest Visual-Visual, lo que corroboraría que la memoria mantiene una interdependencia respecto del desarrollo del lenguaje en el niño.

Finalmente, el estudio sostiene que si se toma en cuenta el control de la variable edad, las correlaciones se vuelven poco significativas, y los puntajes de la Memoria de secuencias no estarían relacionados con la inteligencia "no verbal" medida por el test de la Figura Humana, de allí que se sugiere un mayor control de esta variable demográfica con muestras equivalentes, para estudios posteriores relacionados a la Memoria de Secuencias.

Teniendo en cuenta que se trata de niños cuya personalidad está en formación, donde el componente cognitivo aparece como predominante en los niños de mayor edad, no así en los menores (6 a 9 años) que muestran mayor prevalencia en lo afectivo-emotivo y que se refleja en mayor o menor disposición para el inicio de las tareas.

Tabla N. ${ }^{0}$ 4. Correlaciones parcializadas y sin parcializar por la edad.

\begin{tabular}{ccc}
\hline & $\begin{array}{c}\text { Correlaciones orden } \\
\text { cero }\end{array}$ & $\begin{array}{c}\text { Correlaciones } \\
\text { parcializadas }\end{array}$ \\
\hline Visual-Visual & $0.405^{* *}$ & $0.155 \mathrm{a}$ \\
Visual-Verbal & $0.238^{*}$ & $0.056 \mathrm{a}$ \\
Verbal-Visual & $0.310^{* *}$ & $0.184 \mathrm{a}$ \\
Verbal-Verbal & $0.334^{* *}$ & $0.271 \mathrm{a}$ \\
\hline$* *: p<0.001 ;$ a: no significativo &
\end{tabular}

\section{CONCLUSIONES}

1. No existe relación directa entre las aptitudes cognitivas y las estrategias de memorización de secuencias de los niños de 6 a 11 años.

2. Existe relación directa entre las aptitudes cognitivas y las estrategias de memorización de secuencias, en el subtest de Memoria Visual-Visual de los niños de estas edades, cuando no se controla la variable edad.

3. Existe relación directa entre el menor desempeño en las aptitudes cognitivas y las estrategias de memorización de secuencias, sin mediación verbal, de los niños de estas edades. 


\section{RECOMENDACIONES}

Teniendo en cuenta los hallazgos, consideramos necesario señalar:

1. Que el desarrollo normal del lenguaje es el punto de partida como prerrequisito para la transferencia, en la optimización de las aptitudes cognitivas que se reflejan en los procesos de la memoria de secuencias. De esta capacidad de memoria depende el desarrollo de tareas de mayor complejidad, como aprender a leer y escribir correctamente en los grados escolares, ya que le otorga al niño estrategias psicolinguísticas que, a la postre, le permitirá ser lector experimentado con la suficiente destreza para realizar interacciones afectivas y motivaciones adecuadas en distintos contextos sociales.

2. Tenemos una educación que brinda al niño escasa oportunidad para familiarizarse con el lenguaje escrito y tareas de memorización, descuidando la estimulación necesaria para el desarrollo del pensamiento lógico-simbólico.

Estas carencias no tienen las mismas consecuencias para los niños de clase media urbana en relación con los del contexto urbano-marginales donde el lenguaje que es pobre se refleja en la imaginería, memoria y pensamiento. Tales diferencias se agigantan si la comparación admite a niños del nivel rural, donde el lenguaje restringido puede afectar el proceso de memorización.

De allí que se recomienda que la enseñanza en todos los niveles sea sustentada en la transferencia verbal de la información, para un mejor desempeño escolar.

Finalmente, se debe realizar otros estudios en el medio que favorezcan estrategias de memorización y potencialicen las aptitudes cognitivas en general.

\section{REFERENCIAS BIBLIOGRÁFICAS}

1. Abell, S. C., von Briesen, P. D. \& Watz, L. S. (1996). Intellectual evaluations of children using human figure drawings: an empirical investigation of two methods. Journal of Clinical Psychology 52(1):67-74.

2. Bailey, C.H. y Kandel, E.R. (1986). Aproximaciones moleculares en el estudio de la memoria a corto y a largo plazo. En: Cohen, C.W. (Ed.) Las funciones del cerebro. Barcelona: Ariel.

3. Bauer, R. H. (1977). Memory processes in children with learning disabilities: Evidence for deficiente rehearsal. Journal of Experimental Child Psychology 24, 415-430.

4. Bauer, R. H. (1979). Memory, adquisition, and category clustering in learningdisabled children. Journal of Experimental Child Psychology, 27, 365-383.

5. Bohrnstedt, G. W. (1983). Measurement. En P. H. Rossi, J. D. Wright, \& A. B. Anderson (Eds.) Handbook of Survey Research. San Diego, CA: Academic Press.

6. Ceci, S. J., Ringstrom, M., \& Lea, S. E. G. (1981). Do language learning disabled children (L/LDs) have impaired memories? In Search of Underlying Processes. Journal of Learning Disabilities, 14, 159-163. 
7. Chen, P. Y. \& Popovich, P. M. (2002). Correlation: Parametric and Nonparametric Measures. Thousand Oaks: Sage.

8. Cohen, B. H. (2001). Explaining psychological statistics, 2nd ed. New York: Wiley.

9. Cronbach, L.J. (1951). Coefficient alpha and the internal structure of test. Psychometrika, $16,297-334$.

10. Dawson, M. M., Hallahan, D. R., Reeve, R. E., \& Ball, D. W. (1980). The effects of reinforcement and verbal rehearsal on selective attention in learning disabled children. Journal of Abnormal Child Psychology, 8, 133-144.

11. Fabry, J. J., Bertinetti, J. F. (1990). A construct validation study of the Human Figure Drawing Test. Perceptual and Motor Skills, 70(2):465-6

12. Hebb, D. (1949). The organization of behavior. New York: Wiley.

13. James, W. (1980/1989). Principios de psicología. Fondo de Cultura Económica, México.

14. Kail, R. V. (1984). The development of memory in children (rev. ed.) New York: W.H. Freeman.

15. Lindgren, S. D. \& Richman, L.D. (1984). Inmediate memory functions verbally deficient reading-disabled children. Journal of Learning Disabilities, 17, 222-225.

16. Lindgren, S. D. \& Richman, L. C., \& Eliason, M. L. (1986). Memory processes in reading disability subtypes. Developmental Neuropsychology, 17, 222-225.

17. Lyon, G. R. \& Watson, B. (1981). Empirically derived subgroups of learning disabled readers: Diagnostic characteristics. Journal of Learning Disabilities 17,222-225.

18 . Luria, A. (1980). Neuropsicología de la memoria. Madrid: Blume.

19. Luria, A. R. (1974). Cerebro en acción. Barcelona: Fontanella.

20. Ortiz, C.P. (1998). El nivel consciente de la memoria. Lima: Fondo Editorial de la Universidad de Lima.

21. Ortiz. C. P. (2004). Cuadernos de Psicobiología Social 1. Introducción a la Psicobiología del hombre. Lima: Centro de Producción Editorial e Imprenta de la UNMSM.

22. Ortiz, C. P. (2004). Cuadernos de Psicobiología Social 6. El nivel consciente de la actividad personal. Lima: Centro de Producción Editorial e Imprenta de la UNMSM.

24. Ortiz, C.P. (1994). El sistema de la personalidad. Lima: Centro de Producción Editorial e Imprenta de la UNMSM.

25. Ortiz, C.P. (1996). La formación de la personalidad. Lima: Dimaso Editores.

26. Osborne, J. W. (2003). Effect sizes and the disattenuation of correlation and regression coefficients: lessons from educational psychology. Practical Assessment, 27.

27. Reynolds, C.R. \& Hickman, J. A. (2004). Draw-a-person intellectual ability test for children, adolescents and adults (DAP:IQ). Austin: PRO-ED. Lindgren, S. 
28. D., \& Richman, L. D. (1984). Inmediate memory functions verbally deficient readingdisabled children. Journal of Learning Disabilities, 17, 222-225.

29. Richman, L. C. \& Lindgren, S.D. (1980). Patterns of intellectual ability in children with verbal deficits. Journal of Abnormal Child Psichology, 8, 65-75.

30. Rourke, B. P. (Ed.) (1985). Neuropsychology of learning disabilities. New York: Guilford Press.

31 Sarria, C. Pizarro, P., Llaja, V. (2007). Compendio de Neuropsicología Clínica. Pesquisas Neuropsicológicas. Lima: Fondo Editorial UNMSM.

32. Swanson, L. (1979). Developmental recall lag in learning-disabled children: Perceptual deficit or verbal mediation deficiency? Journal of Abnormal Child Psychology, 7, 199-210.

33. Torgesen, J. K. (1977). Memorization processes in reading disabled children. Journal of Educational Psychology, 69, 571-578.

34. Torgesen, J. K. (1980). Conceptual and educational implications of the use of efficient task strategies by learning disabled children. Journal of Learning Disabilities, 13, 364-371.

33. Torgesen, J. K. (1985). Memory processes in reading disabled children. Journal of Learning Disabilities, 18, 350-357.

34. Torgesen, J. K. \& Dice, C. (1980). Characteristics of research on learning disabilities. Journal of Learning Disabilities, 13, 531-535.

35. Torgesen, J. K. \& Goldman, T. (1977). Verbal rehearsal and short-term memory in reading-disabled children. Child Development, 48, 56-60.

36. Wood, K. M. (1985). Inmediate memory functions in reading disability subtypes. M. A. Thesis Equivalency, University of Iowa, Iowa City.

37. Wood, K., Richman, L. \& Eliason, M. (1989). Inmediate memory functions in reading disability subtypes. Brain and Language, 36, 181-192.

38. Zheng, L. (2000). A Comparing Study between Draw-A-Person Test and CWISC. Chinese Journal of Clinical Psychology, 8(2), 111-113. 